\title{
Device for simultaneous measurement of the Peltier and Seebeck coefticients: Verification of the Kelvin relation
}

\author{
J. Jimenez, E. Rojas, and M. Zamora \\ Departamento de Termología, Universidad de Sevilla, Spain
}

(Received 9 February 1984; accepted for publication 13 June 1984)

We have designed and built an experimental device, which we called a "thermoelectric bridge." Its primary purpose is simultaneous measurement of the relative Peltier and Seebeck coefficients. The systematic errors for both coefficients are equal with this device and manipulation is not necessary between the measurement of one coefficient and the other. Thus, this device is especially suitable for verifying their linear relation postulated by Lord Kelvin. Also, simultaneous measurement of thermal conductivity is described in the text. A sample is made up of the couple nickel-platinum, taking measurements in the range of $-20-60{ }^{\circ} \mathrm{C}$ and establishing the dependence of each coefficient with temperature, with nearly equal random errors $\pm 0.2 \%$, and systematic errors estimated at $-0.5 \%$. The aforementioned Kelvin relation is verified in this range from these results, proving that the behavioral deviations are $\leqslant 0.3 \%$ contained in the uncertainty $\pm 0.5 \%$ caused by the propagation of errors.

\section{INTRODUCTION}

The Seebeck and Peitier effects are associated phenomena, therefore it seems desirable to perform their measurement simultaneously. Standard methods ${ }^{1}$ can be used to measure the Seebeck coefficient with about a $1 \%$ accuracy. The accuracy in measuring the Peltier coefficient is somewhat less because the Peltier and Joule effects take place simultaneously and the difficulty in measuring heat flux is enhanced by the necessity to separate the contribution of each effect. ${ }^{2}$ Previously, because both coefficients have been determined by different experimental procedures, and the results have been obtained with different accuracy, the verification of the Kelvin relationship has been difficult and ambiguous. This relation $(\Pi=S T, \Pi$ is the Peltier coefficient, $S$ is the Seebeck coefficient, and $T$ is the temperature) was proven with an uncertainty of between $5 \%$ and $10 \%$, and as far as we know, has not been improved.

This paper presents a new experimental device which permits us to perform measurements of the relative Seebeck and Peltier coefficients. Its most useful characteristic is that the sample and the remaining system do not need to be handled between both types of measurements. Moreover, in both cases the random errors are nearly equal and the systematic ones, specific for the device, are theoretically equal and consequently they do not propagate to the Kelvin relation. For metals, the sample is in the form of a thermopile using the chosen pair. Therefore, the thermoelectric effects will be multiplied by the number of thermocouples which form the thermopile. This fact allows us to operate with significant outputs closer to thermal equilibrium. The basic elements of the device are platinum resistors and thermopiles, which fulfill dual functions, sometimes as measurers of heat flux or temperature, other times as heaters or coolers, depending on the process of calibration or measurement. The parameters determining the device, including the sample, are strongly coupled and fixed using criteria (see Appendix $B$ ) in order to enhance the system outputs, to minimize the experimental errors, and reach a certain symmetry which facilitate the measurements. Although the device has been designed to work with metal samples, it is easily applicable to other substances, such as semiconductors, for example.

With the purpose of checking the device and testing the method of measurement we have made experiments with a sample thermopile of the couple $\mathrm{N}$ - $\mathrm{Pt}$.

\section{EXPERKMENTAL- DEVICE}

The experimental device consist of three thermopiles $\left(\phi_{1}, \phi_{2}\right.$, and $\left.\phi_{s}\right)$, three platinum electrical resistances $\left(t_{1}, t_{2}\right.$, and $\left.t_{s}\right)$, and a heat reservoir $H$. The thermopiles are aligned (see Fig. 1) so that $\phi_{s}$ is placed between $\phi_{1}$ and $\phi_{2}$ whose free

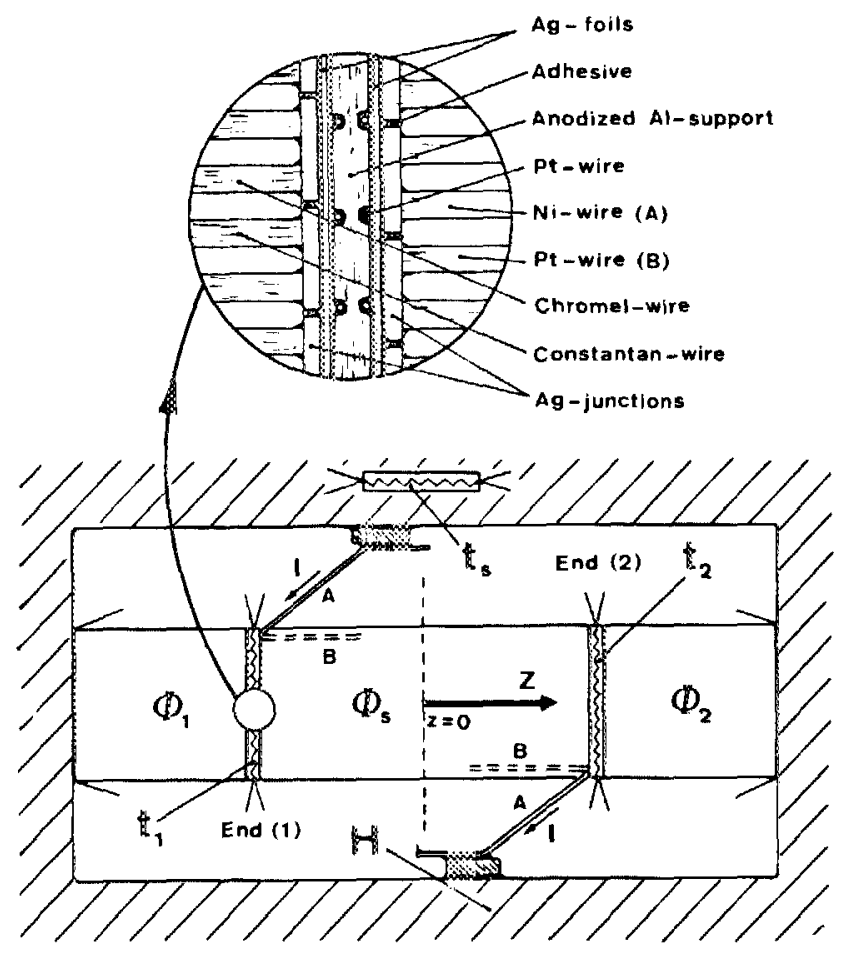

FIG. 1. Schematic side view of the "thermoelectric bridge," described in Sec. II. The insert shows a cross section. 
ends are in thermal contact with the heat reservoir. The resistances $t_{1}$ and $t_{2}$ are located on either end of the thermopile $\phi_{s}$ and between thermopiles $\phi_{1}$ and $\phi_{2}$. The resistance $t_{s}$, which is a commercial platinum thermometer (Leeds \& Northrup, mod. $8164 \mathrm{~B}$ ) is lodged in the heat reservoir. These thermopiles are made with a technique we have described in another paper. ${ }^{4}$

We shall perform two operations with the device. First, with $\phi_{1}$ and $\phi_{2}$ we can measure the different heat fluxes between the ends of $\phi_{s}$ and the heat reservoir. These heat fluxes are produced by thermal asymmetry, due to the Peltier effect, when a continuous electrical current goes through $\phi_{s}$. We shall determine the value of the relative Peltier coefficient of the couple constituting $\phi_{s}$ in terms of the values of those heat fluxes, the applied electrical current, and the specific parameters of the device. Secondly, we can produce, via the Peltier effect, a similar thermal asymmetry to the above, but now injecting the electrical current into $\phi_{1}$ and $\phi_{2}$. Thus, we shall determine the relative Seebeck coefficient of the same couple in terras of the emf generated by $\phi_{s}$, and the temperature difference between these ends, measured by $t_{1}$ and $t_{2}$. In conclusion, we generate antisymmetrical effects in a symmetric device, and detect them with symmetric measurers. We believe it would not be improper to call the above described device a "thermoelectric bridge."

The sample thermopile $\phi_{s}$ built to verify our system, is made up of 11 nickel-platinum thermocouples (both of $99.99 \%$ purity). The thermal resistance between their ends is about $100 \mathrm{~K} / \mathrm{W}$, at room temperature. Their electrical terminals, made of nickel, come out of each end to be thermally anchored at the heat reservoir.

The thermopiles $\phi_{1}$ and $\phi_{2}$ are equal and are composed of 50 chromel-constantan thermocouples. The electrical terminals, made of copper, come out of the end in thermal contact with the heat reservoir. The thermal resistance of each one is about $50 \mathrm{~K} / \mathrm{W}$ at room temperature.

The electrical resistances $t_{1}$ and $t_{2}$ are equal and are supported on two anodized aluminium disks, $0.5 \mathrm{~mm}$ in thickness, with narrow channels engraved on their sides. A platinum wire, with a nominal resistance of $20 \Omega$, is held in place in these channels by a high thermal conductivity adhesive. A more detailed description of these devices may be found in another paper. ${ }^{4}$ During the experiments, these resistances will sometimes measure the temperature at the ends of $\phi_{s}$, and at other times dissipate heat power of known value.

The heat reservoir consists' of a $10-\mathrm{kg}$ bronze block surrounded by two radiation shields and confined in a vacuum chamber, which is placed in a thermostat.

The geometrical parameters of the above names thermopiles and resistances are fixed applying the criteria described in Appendix B. These elements have the same section $1 \mathrm{~cm}^{2}$ perpendicular to the $z$ axis; see Fig. 1. They are lodged in the bronze block by means of an intermediate bronze container, which is not drawn in the diagram. This container facilitates the setting up, maintains their alignment, and establishes the thermal contacts (assisted by berylium oxide and silicone) among the different elements. Extreme precautions are taken to achieve symmetry in the device about the plane $z=0$, and a one-dimensional temperature gradient in the device. For isolation conditions, vacuum is maintained about $10^{-6}$ Torr.

The calibration of the elements of the thermoelectric bridge, in order to convert the thermopiles to heat fluxmeters ${ }^{4}$ and the electrical resistances to thermometers, is performed when these are set in the device. To calibrate $\phi_{1}$ and $\phi_{2}$, known heat fluxes are produced by dissipating Joule heat power alternately at $t_{1}$ and $t_{2}$. When a heat power $W$ is dissipated at $t_{1}$ and the steady state has been reached, two heat fluxes, $f_{1}$ and $f_{2}$, start from here towards both directions of the $z$ axis in Fig. 1 . The heat flux $f_{1}$ passes through $\phi_{1}$ while $f_{2}$ passes through $\phi_{2}$. Calling the emf's produced by these fluxes at $\phi_{1}$ and $\phi_{2}$ respectively, $E_{1}$ and $E_{2}$, and $k_{1}=f_{1} / E_{1}$ and $k_{2}=f_{2} / E_{2}$ being the corresponding calibration parameters, we can write $W=k_{1} E_{1}+k_{2} E_{2}$. Now dissipating $W^{\prime}$ at $t_{2}$, we obtain $W^{\prime}=k_{1} E_{1}^{\prime}+k_{2} E_{2}^{\prime}$, with $E_{1}^{\prime}$ and $E_{2}^{\prime}$ being the emfs measured on $\phi_{1}$ and $\phi_{2}$, respectively. Solving the above equations we obtain the values of $k_{1}$ and $k_{2}$. This process is repeated for all operating temperatures to obtain the fits $k_{1}(T)$ and $k_{2}(T)$, which allow us to calculate the heat fluxes as a function of the emf's they generate. The electrical resistances, $t_{1}$ and $t_{2}$ are calibrated against the commercial thermometer $t_{s}$, lodged in the thermal reservoir, by measuring their values, $R_{1}$ and $R_{2}$ at different temperatures, and establishing the fits $R_{1}(T)$ and $R_{2}(T)$. The results of calibration are given in Appendix A.

By a combination of the operations described in the second paragraph of this section, the thermoelectric bridge is suitable to measure thermal conductivity. This is performed as follows. One of the thermopiles act as heater or cooler, via Peltier effect, creating a one-dimensional temperature gradient in the sample, while the other thermopile measures the corresponding heat flux. At the same time, the thermometers $t_{1}$ and $t_{2}$ measure the temperature difference between the ends of the sample.

Our experience $^{5}$ is that it is easy to achieve the desired temperature differences and their stability. The characteristics $^{4}$ of the used thermometers and thermopiles make them especially suitable for this type of measurement.

For samples made with a single couple device (semiconductor platinum-plated at its ends, for example), Seebeck and Peltier coefficients, and thermal conductivity can be simultaneously measured.

\section{PRINCIPLES OF MEASUREMENT}

To express the relative Peltier coefficient of the couple constituting $\phi_{s}$ as a function of measurable quantities, we need to consider the separability of the various types of heat generated when a continuous electric current is supplied to $\phi_{s}$. This separability is based on the nature of the thermal effects and the symmetry of the thermoelectric bridge. The heat dissipated along the wires of $\phi_{s}$, including its terminals, is caused by Joule and Thomson effects. Heats of different sign due to the Peltier effect are dissipated at the ends of $\phi_{s}$, as well as Joule heats associated with the electrical resistance of the junctions. Near thermal equilibrium, the Peltier heat generation will be antisymmetric with regard to the plane $z=0$ in Fig. 1, while the Joule heat generation will be sym- 
metric, if the symmetry of the bridge is precise. The Thomson heat generation, several orders of magnitude less than the others, can be considered nearly symmetric, since the temperature distribution along the wires is nearly linear in our working conditions. Thus, the total heat power $W_{J+T}$ dissipated in the wires of $\phi_{s}$ produces equal heat fluxes of value $W_{J+T} / 2$ at its ends.

When a steady state is reached, the heat fluxes at the ends of $\phi_{s}$ (assuming that all of them come out of the ends of $\left.\phi_{s}\right)$ are

through the fluxmeters

inside of $\phi_{s}$

End (I): $f_{1}=-\frac{T_{0}-T_{1}}{r}, \quad q_{1}=-\frac{T_{2}-T_{1}}{r_{s}}-\frac{W_{J+T}}{2}$,

End (2): $f_{2}=-\frac{T_{0}-T_{2}}{r}, \quad q_{2}=-\frac{T_{1}-T_{2}}{r_{s}}-\frac{W_{J+T}}{2}$,

where $T_{0}$ is the heat reservoir temperature, $T_{1}$ and $T_{2}$ are the temperatures of the ends of $\phi_{s}, r$ is the thermal resistance of each fluxmeter, and $r_{s}$ is the thermal resistance of $\phi_{s}$.

According to the direction of the current in Fig. 1, the equations of energy balance, between the powers dissipated at the ends of $\phi_{s}$ and the heat fluxes departing there, are

$$
\begin{aligned}
& \text { End (1): }-N \Pi_{A B}\left(T_{1}\right) I+R I^{2}=f_{1}+q_{1}, \\
& \text { End (2): }-N \Pi_{B A}\left(T_{2}\right) I+R I^{2}=f_{2}+q_{2},
\end{aligned}
$$

where $I$ is the applied current, $\Pi_{A B}$ is the Peltier coefficient of the couple $A-B, N$ is the number of thermocouples of $\phi_{s}$, and $R$ is the electrical resistance of the junctions at each end.

Inserting the expressions of $q_{1}$ and $q_{2}$ from Eq. (1) into Eqs. (1) and (2), and subtracting the resulting expressions, taking into account $T_{2}-T_{1}=\left(f_{2}-f_{1}\right) r$, we obtain,

$$
2 N \Pi_{A B}(\bar{T}) I=(2 A+1)\left(f_{2}-f_{1}\right)
$$

where $\bar{T}=\left(T_{2}+T_{1}\right) / 2$ (which in our operating conditions is nearly equal to $\left.T_{0}\right)$ and $A=r / r_{s}$.

Performing a more realistic analysis, where the symmetry of the thermoelectric bridge is not entirely reached, we obtain,

$$
2 N \Pi_{A B}\left(T_{0}\right) I+\delta R I^{2}=2\left(f_{2} A_{2}-f_{1} A_{1}\right)+\left(f_{2}-f_{1}\right),
$$

where $A_{1}=r_{1} / r_{s}$ and $A_{2}=r_{2} / r_{s}$, being $r_{1}$ and $r_{2}$ the thermal resistances of $\phi_{1}$ and $\phi_{2}$ respectively (see Appendix A), and $\delta R$ denotes the asymmetry in the distribution of the electrical resistance of $\phi_{s}$.

The expression of the Seebeck coefficient of the sample couple is $S_{A B}=E_{\mathrm{s}} / N \Delta T$, where $E_{s}$ is the emf generated by $\phi_{s}$, and $\Delta T$ is the temperature difference applied between its ends. In our operating conditions $\Delta T$ can be small enough to apply the linear approximation.

\section{SYSTEMATIC ERRORS}

One cause of error is a possible defect in the symmetry intended for the bridge. As we have seen, symmetry with resepct to plane $z=0$ in Fig. 1 allows us to separate the different thermal effects. The only term which is not directly measurable in Eq. (5), $\delta R I,{ }^{2}$ comes from a possible asymmetry but is independent of the inversion of the current, and can thus be removed. Two different temperature distributions are generated by means of this reversal. To make these distributions nearly enantiomorph with regard to the plane $z=0$ so that the results are comparable, it is necessary to come as close as possible to this symmetry. Fortunately we can verify the intended symmetry by means of the results of calibration and the response of the fiuxmeters when an alternating electrical current is injected into $\phi_{s}$.

Other important causes of errors proceed from the quality of the thermal contact between the different elements of the bridge. In particular, the small thermal resistances between $t_{1}$ and $t_{2}$ and the ends of $\phi$ generate systematic errors. Provided that the symmetry is perfect and $\delta r$ denoting each small thermal resistance, the value of $A$ in Eq. (4) is $(r+\delta r) / r_{s}$, while the value obtained in the calibration corresponds to $r /\left(r_{s}+2 \delta r\right)$, since in the latter case the heat is dissipated in the thermometers. This fact yields a systematic relative error $-2 \delta r / r_{s}$ in the Peltier coefficient. During the measurement of the Seebeck coefficient the temperature differences between the ends of $\phi_{s}$ are not measured at these ends, but at the thermometers, which are separated from them by the same thermal resistance, $\delta r$. Because of this fact, another relative systematic error, also equal to $-2 \delta r / r_{s}$ is generated. Equal systematic error corresponds to thermal conductivity measurement.

Errors by heat leaks through electrical terminals are not produced by this device. ${ }^{4}$

\section{RESULTS AND DISCUSSION}

Measurements are made in the range of $-20-60^{\circ} \mathrm{C}$ with steps of about $20^{\circ} \mathrm{C}$. The different electric currents applied are measured using standard resistances inserted in series in the supply devices, in such a way that the input as well as the output of the system can be measured with a voltmeter, in our case Keithley 181. Every mobile electric connection is made with amalgamated copper, and all of the input and output devices, thermostated at $21.5 \pm 0.2^{\circ} \mathrm{C}$, are placed in a Faraday shield. The time necessary to reach the steady state in the thermoelectric bridge is about $400 \mathrm{~s}$ for all processes.

Using the method described in Sec. IIII, we supply a continuous electric current to $\phi_{s}$ and, when a steady state is reached, we measure the voltage output of the fluxmeters, convert them to the fluxes $f_{1}$ and $f_{2}$, and introduce these values in Eq. (5) along with those of the calibration parameters $A_{1}, A_{2}$ corresponding to the temperature of the experiment. In order to remove from Eq. (5) the term corresponding to a possible asymmetry in the Joule heat generation, the experiment is repeated reversing the electric current. For each temperatire at the thermal reservoir, this operation is carried out about ten times by changing the current in the range $40-140 \mathrm{~mA}$. The highest extreme corresponds to the so-called "inversion current" (see Appendix B) and the lowest one is fixed to obtain heat fuxes of the same order in all the experiments. For each thermal reservoir temperature we plotted the value of the power $2\left(f_{2} A_{2}-f_{1} A_{1}\right)+\left(f_{2}-f_{1}\right) / 2 N$ [see Eq. (5)] versus the supplied current, and the value of the slope $m$ of the straight line is taken as the Peltier coefficient. The results obtained and data characterizing the fits are pre- 
TABLE I. Values of the relative Peltier coefficient of the Ni-Pt sample at each work temperature and data characterizing the linear fits.

\begin{tabular}{rcccc}
\hline \hline $\begin{array}{c}\text { Temperature } \\
\left({ }^{\circ} \mathrm{C}\right) \\
\pm 0.1^{\mathrm{s}}\end{array}$ & No. & $\begin{array}{c}\Pi_{\mathrm{Ni}-\mathrm{Pt}}{ }^{\mathrm{b}} \\
(\mathrm{mV})\end{array}$ & $\begin{array}{c}\text { (m) } \\
(\mathrm{mV})\end{array}$ & C.C. \\
\hline-19.4 & 20 & 3.225 & \pm 0.001 & 0.999999 \\
0.1 & 16 & 3.592 & \pm 0.002 & 0.999998 \\
25.2 & 22 & 4.091 & \pm 0.003 & 0.999996 \\
40.1 & 22 & 4.380 & \pm 0.003 & 0.999995 \\
57.6 & 22 & 4.729 & \pm 0.004 & 0.999995 \\
\hline
\end{tabular}

"Corresponding to the maximum fluctuation of the average temperature of the sample for each set of measurements.

${ }^{b}$ Equal to the slope (systematic errors are not included).

Key: No. $=$ number of measurements, $\epsilon(m)=$ uncertainty of the slope, C.C. $=$ correlation coefficient.

sented in Table I. We show in Fig. 2 the Peltier coefficient versus temperature, and the best fitting curve is

$$
\Pi(T)=3.595+0.0193 T+0.7 \times 10^{-5} T^{2},
$$

$\Pi(T)$ is expressed in $\mathrm{mV}$ and $T$ in ${ }^{\circ} \mathrm{C}$. The standard deviation is 0.005 . Moreover, we must keep in mind the systematic error, estimated at $-0.5 \%$, which arises from the aforementioned dissimilarity between the calibration and measurement conditions.

A similar procedure to the above one is performed, but now the electric current is supplied to thermopiles $\phi_{1}$ and $\phi_{2}$, so that, due to the Peltier effect, the required temperature difference between the ends of $\phi_{s}$ is obtained. As soon as the steady state is reached, this difference and the corresponding voltage output of $\phi_{s}$ are annotated. For each temperature at the thermal reservoir, the measurement is performed for about ten different values of electrical current in both directions (to remove spurious emf's by average procedure) so that the temperature difference changes from $\pm 0.5-$ $\pm 3.5^{\circ} \mathrm{C}$. The Seebeck coefficient, at each temperature, is obtained from the slope $m$ of the plot of $E_{s} / 11$ versus temperature difference between the ends of $\phi_{s}$. The results obtained and data characteristics of the fits are presented in Table II. We show in Fig. 3 the Seebeck coefficient versus

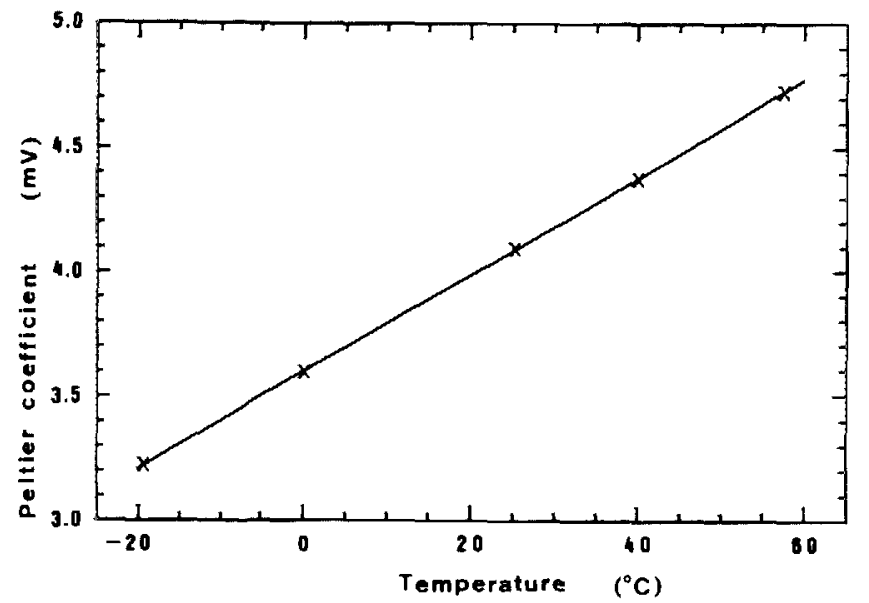

FIG. 2. Relative Peltier coefficient of Ni-Pt vs temperature.
TABLE II. Values of the relative Seebeck coefficient of the Ni-Pt sample at each work temperature and data characterizing the linear fits.

\begin{tabular}{rcccc}
\hline $\begin{array}{c}\text { Temperature } \\
\left({ }^{\circ} \mathrm{C}\right)\end{array}$ & No. & $\begin{array}{c}S_{\text {Ni-Pt }}{ }^{\mathrm{b}} \\
(\mu \mathrm{V} / \mathrm{C})\end{array}$ & $\begin{array}{c}\epsilon(m) \\
(\mu \mathrm{V} / \mathrm{C})\end{array}$ & C.C. \\
\hline$-18.8 \pm 0.2^{\mathrm{n}}$ & 12 & 12.746 & \pm 0.008 & 0.999998 \\
$0.8 \pm 0.2$ & 20 & 13.174 & \pm 0.005 & 0.999998 \\
$25.8 \pm 0.3$ & 22 & 13.722 & \pm 0.006 & 0.999998 \\
$40.8 \pm 0.2$ & 20 & 13.963 & \pm 0.004 & 0.999999 \\
$58.2 \pm 0.2$ & 18 & 14.267 & \pm 0.006 & 0.999998 \\
\hline
\end{tabular}

- Corresponding to the maximum fluctuation of the average temperature of the sample for each set of measurements.

${ }^{b}$ Equal to the slope (systematic errors are not included).

Key: No. $=$ number of measurements, $\epsilon(m)=$ uncertainty of the slope, C.C. $=$ correlation coefficient.

temperature and the best fitting curve is

$$
S(T)=13.170+0.022 T-5.5 \times 10^{-5} T^{2},
$$

$S(T)$ is expressed in $\mu \mathrm{V} /{ }^{\circ} \mathrm{C}$ and $T$ in ${ }^{\circ} \mathrm{C}$. The standard deviation is 0.02 . Here again it is necessary to consider a systematic error equal to $-0.5 \%$. We note how easy it is to impose the required temperature differences. The stability of these differences is verified by the corresponding stability of the voltage output of $\phi_{s}$, whose fluctuations do not surpass the noise of the voltmeter used.

We use the expressions (6) and (7) to verify, without ambiguity, the second Kelvin relation in the working temperature range. For this purpose we define the function,

$$
2(I I-S T) /(I I+S T),
$$

which expresses a possible behavioral deviation. In Fig. 4, this function is plotted versus temperature. It can be seen that the deviation remains less than $0.3 \%$, which is less than the uncertainty $0.5 \%$, corresponding to error propagation.

Although the degree of certainty with which this relationship is verified is very high, the working temperature range is narrow. We are currently modifying the system for measurements over a considerably broader temperature range.

\section{APPENDIX A: CALIBRATION}

Calibration of the thermopiles, to convert them to heat flux measurers, is performed at each of the temperatures

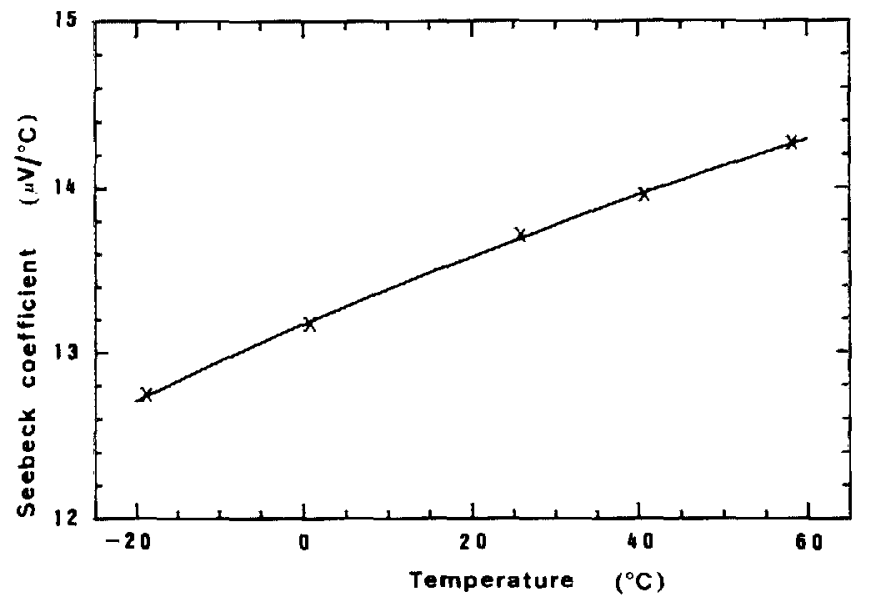

FIG. 3. Relative Seebeck coefficient of Ni-Pt vs temperature. 


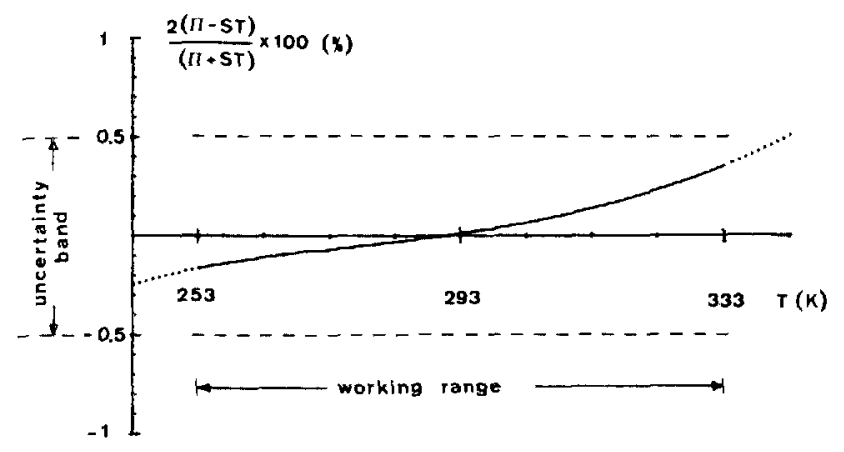

FIG. 4. Behavioral deviation of the Kelvin relation vs temperature.

where a measurement is to be taken. We obtain the values of the calibration parameters, $k_{1}$ and $k_{2}$, by means of the method exposed in Sec. II. The dissipated powers are chosen so that the temperature differences between the ends of the thermopiles are of the same order as the ones in the measurement of the Peltier coefficient (about $0.1^{\circ} \mathrm{C}$ ). For the results obtained, the best fittings are

$$
\begin{aligned}
& k_{1}(T)=7.312+1.7 \times 10^{-4} T+4.5 \times 10^{-5} T^{2}, \\
& k_{2}(T)=7.476-3.8 \times 10^{-4} T+5.0 \times 10^{-5} T^{2} .
\end{aligned}
$$

The calibration parameters are expressed in $\mathrm{W} / \mathrm{V}$ and $T$ in ${ }^{\circ} \mathrm{C}$. The standard deviations for both parameters are equal to $4 \times 10^{-3}$.

The values of the parameters $A_{1}$ and $A_{2}$ (see Sec. III) and their dependence on temperature are obtained simultaneously with the calibration of the heat fluxmeters by considering the temperature distributions in the thermoelectric bridge when heat powers are dissipated in $t_{1}$ and $t_{2}$ and applying the Fourier law for heat conduction. When $W$ is dissipated in $t_{1}$ we have $k_{1} E_{1} r_{1}=k_{2} E_{2}\left(r_{s}+r_{2}\right)$, and when $W^{\prime}$ is dissipated in $t_{2}$ we have $k_{2} E_{2}^{\prime} r_{2}=k_{1} E_{1}^{\prime}\left(r_{s}+r_{1}\right)$. Substituting the parameters $k_{1}$ and $k_{2}$ for their expressions as a function of the applied powers and resulting emfs in the fluxmeters we obtain $A_{1}=W^{\prime} E_{2} /\left(W E_{2}^{\prime}-W^{\prime} E_{2}\right)$ and $A_{2}=W E_{1}^{\prime} /\left(W^{\prime} E_{1}-W E_{i}^{\prime}\right)$. Then we perform the fits $A_{1}(T)$ and $A_{2}(T)$ starting from the values obtained at all operating temperatures,

$$
\begin{aligned}
& A_{1}(T)=0.6780-1.69 \times 10^{-3} T+1.4 \times 10^{-6} T^{2}, \\
& A_{2}(T)=0.6615-1.67 \times 10^{-3} T+2.0 \times 10^{-6} T^{2},
\end{aligned}
$$

$T$ is expressed in ${ }^{\circ} \mathrm{C}$ and the standard deviations are $5 \times 10^{-4}$ for $A_{1}$ and $6 \times 10^{-4}$ for $A_{2}$.

These results demonstrate that the intended symmetry is well attained.

In the calibration of thermometers $t_{1}$ and $t_{2}$, it is only worth commenting on the systematic error because of the location of the thermometers in the experimental device. A thermopile which has a thermal resistance of about $50 \mathrm{~K} / \mathrm{W}$ is placed between each thermometer and the thermal reservoir. Because a power of about $2 \times 10^{-5} \mathrm{~W}$ is dissipated in each thermometer in a steady state, a temperature difference of $1 \mathrm{mK}$ (in the working range) arises between each thermometer $t_{1}$ and $t_{2}$ and the reference thermometer $t_{s}$. This fact is detected by measuring the voltage output of the fluxmeters. Due to the symmetry of the thermoelectric bridge, these temperature differences are equal, which is verified by the zero voltage output of $\phi_{s}$ during this process. Therefore, they do not influence the results of the Seebeck coefficient measurements.

\section{APPENDIX B: PERFORMARCE}

To perform measurements near equilibrium, we design the bridge in such a way that the temperature difference between the ends of $\phi_{s}$ for the applied current and the electrical response corresponding to the difference are the greatest.

With regard to the Peltier effect, inserting the expressions of $f_{1}$ and $f_{2}$ from Eq. (1) in Eq. (4), we obtain

$$
\frac{T_{1}-T_{2}}{I}=2 N \Pi_{A B} \frac{r r_{s}}{r_{s}+2 r} .
$$

This function increases indefinitely as $r$ and $r_{s}$. However, for $r_{s}+2 r=$ constant, it passes through a maximum if $r_{s}=2 r$ or $N / r_{A B}=n / 2 r_{a b}$, where $n$ is the number of thermocouples of each fluxmeter, $r_{A B}$ is the thermal resistance of each thermocouple of $\phi_{s}$, and $r_{a b}$ is the thermal resistance of each thermocouple of the fuxmeters. Making the bridge with this maximum thermal response condition, we obtain

$$
\frac{T_{1}-T_{2}}{I}=\Pi_{A B} \frac{r_{A B}}{2},
$$

which is independent of the number of thermocouples. Nevertheless, the electrical response to this difference is

$$
\frac{E_{1}-E_{2}}{T_{1}-T_{2}}=n S_{a b},
$$

where $S_{a b}$ is the relative Seebeck coefiicient of the couple forming the fluxmeters. This electrical response does increase as the number of thermocouples of the fluxmeters. [The thermoelectric bridge is analogous to an electrical low filter with $Z(w=0)=S_{a b} \Pi_{A B} N r_{a b}=S_{a b} \Pi_{A B} n r_{A B} / 2$.]

Obviously, in the measurement of the Seebeck coefficient, the electrical response of $\phi_{s}$ to a temperature difference between its ends also increases as the number of thermocouples constituting it.

It is desirable to attain elevated densities of the junctions at the ends of all the thermopiles so as not to yield significant thermal inhomogeneities at these ends. Moreover, this condition gives mechanical consistence to the thermopiles, which can thus support the pressure necessary to establish good thermal contacts.

We also take into account the magnitude of the Joule effect which arises during the measurement of the Peltier coefficient. Analyzing the temperature at each end of $\phi_{s}$ (taking into account the condition $r_{s}=2 r$ ) we obtain temperature differences with regard to the thermal reservoir equal to $\pm N I I_{A B} r_{s} I / 4+r_{s} R_{s} I^{2} / 4$, where $R_{s}$ is the electrical resistance of $\phi_{s}$. The first term is due to the Peltier effect and the second one is caused by the Joule effect. We call the electrical current for which the "cold end" of $\phi_{s}$ maintains the same temperature as the thermal reservoir "inversion current"; $I=\Pi_{A B} / R_{A B}$, where $R_{A B}$ denotes the electrical resistance of each thermocouple of $\phi_{s}$ [We shall always adopt this as the maximum value of the applied current. Above it, the Joule effect begins to dominate over the Peltier effect and the ap- 
proximations made to obtain Eq. (5) lose validity.]

The specific design for the chosen sample is explained in Sec. II. We have taken into account the limitations and technological possibilities, as well as the range and accuracy of the measuring instruments to be used. First, we have fixed limits of error for the results and then, through successive approximations under the criteria mentioned, we have deter- mined the values of the system's parameters.

${ }^{1}$ R. D. Barnard, Thermoelectricity in Metals and Alloys (Taylor \& Francis, London, 1972), Chap. 2.

${ }^{2}$ Charles A. Domenicali, Rev. Mod. Phys. 26, 237 (1954).

${ }^{3}$ G. Borelius, Ann. Phys. (Paris) 52, 398 (1917); 56, 388 (1918).

${ }^{4} J$. Jiménez, E. Rojas, and M. Zamora, J. Appl. Phys. 56, 3353 (1984).

5J. Jiménez, Dr. thesis, Universidad de Sevilla (1982). 